\title{
Efficacy of a new prescription-only medical food supplement in alleviating signs and symptoms of dry eye, with or without concomitant cyclosporine $\mathrm{A}$
}

\author{
This article was published in the following Dove Press journal: \\ Clinical Ophthalmology \\ 24 August 2011 \\ Number of times this article has been viewed
}

\author{
Mitchell A Jackson' \\ Kim Burrell ${ }^{2}$ \\ Ian B Gaddie ${ }^{3}$ \\ Shana D Richardson ${ }^{4}$ \\ 'Jacksoneye, Lake Villa, IL, USA; \\ ${ }^{2}$ Centennial Eye Associates, \\ Centennial, CO, USA ${ }^{3}$ Gaddie \\ Eye Centers, Louisville, KY, USA; \\ ${ }^{4} \mathrm{CYNACON} / \mathrm{OCuSOFT}$, \\ Richmond, TX, USA
}

Correspondence: Mitchell A Jackson Jacksoneye, 300 N Milwaukee Ave, Suite L, Lake Villa, IL 60046, USA

$\mathrm{Tel}+$ I 847445549 I

Fax + I 8473560757

Email mjlaserdoc@msn.com
Purpose: To evaluate the effect of a new, prescription-only medical food supplement containing omega-3 and omega- 6 essential fatty acids on dry eye signs and symptoms, with or without concomitant topical cyclosporine.

Methods: A total of 43 subjects were randomized and followed for 6 months. Group $1(n=23)$ was assigned to take two soft geltabs of the medical food supplement by mouth twice daily for 6 months. Group $2(n=20)$ was directed to take the medical food supplement in the same manner, along with topical cyclosporine, instilled twice daily during the last 3 months of the study. Subjects were evaluated at baseline, month 1, month 3, and month 6. Primary outcome measures included tear breakup time (TBUT), conjunctival staining, corneal staining, and change in subjective symptoms.

Results: Both groups had a statistically significant improvement in TBUT between baseline and month 6 . In the food supplement only group, TBUT improved by 0.805 seconds from baseline to month 6 . In the supplement/cyclosporine group, TBUT improved by 1.007 seconds from baseline. There was no statistically significant difference in TBUT between the two groups at baseline, month 3 , or month 6 . There were no significant differences in corneal or conjunctival staining between or within groups. Subjective symptoms were also improved in both groups.

Conclusion: Supplementation with the proper balance of omega- 3 and omega- 6 essential fatty acids improved TBUT and relieved patient symptoms. The addition of topical cyclosporine did not convey any statistically significant improvement in TBUT beyond that achieved by the supplement.

Keywords: omega-3, omega-6, essential fatty acids, TBUT, tear breakup time

\section{Introduction}

Dry eye syndrome is a multifactorial disease affecting the ocular surface. A key mechanism in the initiation of dry eye is tear hyperosmolarity, which then activates a cascade of inflammatory events on the ocular surface and in the tears, contributing to a cycle of inflammation of the lacrimal gland, instability of the tear film, and worsening symptoms. ${ }^{1,2}$

Between $14 \%$ and $33 \%$ of the population worldwide suffers from dry eye, making it a significant public health problem. ${ }^{1}$ Dry eye symptoms, which include discomfort and visual blurring, account for nearly half of all primary or secondary eye care complaints. 
Risk factors that have been closely associated with dry eye include increasing age, female gender, androgen deficiency, hormone replacement therapy, systemic antihistamine use, connective tissue disease, history of refractive surgery, vitamin A deficiency, and low dietary intake of omega-3 and omega- 6 fatty acids. ${ }^{3}$

An International Task Force (ITF) Delphi panel recommended a 4-point severity scale for dry eye (which they termed "dysfunctional tear syndrome"), ranging from mild/ episodic (level 1) to severe (level 4). ${ }^{3}$ This scale was adopted by the International Dry Eye Workshop (DEWS), which added treatment recommendations. For level 2 severity (moderate dry eye with no corneal involvement and mild to no conjunctival staining) and above, the DEWS report recommends one or more of the following treatments: unpreserved tears, gels, ointments, cyclosporine A (Restasis ${ }^{\circledR}$ ) (Allergan, Irvine, CA), secretagogues, topical steroids, and nutritional support (omega-3 and omega-6 essential fatty acids [EFAs]). ${ }^{4}$ Additional interventions are recommended as signs and symptoms worsen at severity levels 3-4.

Restasis therapy, along with artificial tears, has become the leading treatment for patients with dry eye. Cyclosporine is an immunomodulator with anti-inflammatory effects. In the clinical trials that led to the United States Food and Drug Administration's approval of Restasis, 59\% of subjects achieved improvement from baseline Schirmer scores at 6 months, ${ }^{5}$ and additional clinical experience has subsequently demonstrated the benefits of treatment with topical cyclosporine.

Dietary supplementation with EFAs is among the treatments suggested by the expert panels because research has shown a positive effect on dry eye. ${ }^{6}$

Omega-3 and omega- 6 polyunsaturated EFAs are precursors of lipid mediators known as eicosanoids that play an important role in regulating inflammation. Eicosanoids derived from omega-3 are anti-inflammatory, while those derived from omega- 6 are generally considered to be proinflammatory. Supplementation or increased dietary intake of foods containing omega-3 EFAs has been recommended, for a range of health benefits, to balance out the more common omega-6 EFAs in the typical Western diet.

In the ocular setting, higher intake of omega-3 EFAs has been associated with decreased risk of age-related macular degeneration ${ }^{7}$ and dry eye. ${ }^{8}$ Studies have suggested that nutritional supplementation with omega-3 fatty acids is related to improvement in dry eye and may contribute to restoration of the lipid layer, decreased inflammation and apoptosis, and increased tear secretion..$^{9-11}$ A recently published pilot study found that average tear production and tear volume was increased in a group of patients taking an omega-3 supplement containing both fish oil and flaxseed oil. ${ }^{12}$

The balance between omega- 3 and omega- 6 EFAs is important because they work synergistically. ${ }^{10}$ In fact, rat models have suggested that supplements which include a combination of omega-3 and omega-6 EFAs may be ideal. ${ }^{13,14}$ Although omega-6 EFAs are considered pro-inflammatory, certain plant-based omega-6 EFAs, such as gamma-linolenic acid (GLA), also have anti-inflammatory properties. According to Belch and Hill GLA elevates dihomo-g-linolenic acid concentrations, leading to an increased synthesis of 1 -series prostaglandins that have a negative feedback role in chronic inflammation. ${ }^{15}$

A number of studies have shown a relationship between certain plant-based omega-6 EFA supplementations and the improvement of signs and symptoms in ocular surface disease, including contact lens-associated dry eye, ${ }^{16}$ meibomian gland dysfunction, ${ }^{17}$ and Sjögren's syndrome. ${ }^{18}$ Therapy with plant-based omega-6 and artificial tears has also been shown to reduce conjunctival inflammation in dry eye patients. ${ }^{19}$

This study was designed to evaluate the effect of a new, prescription-only medical food supplement containing omega-3 and plant-based omega-6 EFAs on dry eye signs and symptoms, with or without concomitant topical cyclosporine.

\section{Materials and methods}

This was a prospective, randomized trial conducted at two clinical sites.

To be enrolled in the study, patients had to have a diagnosis of ITF level 1 dry eye or worse, based on subjective symptoms and at least one of three clinical signs of dry eye: Sodium fluorescein corneal staining; lissamine green conjunctival staining; and/or tear breakup time (TBUT) $<3$ seconds. Symptoms were elicited via a standardized Ocular Surface Disease Questionnaire (Paul M Karpecki, OD, Karpecki Resource Group, Lexington, KY).

Exclusion criteria included: use of any topical therapeutic ocular medications within 7 days or any anti-inflammatory medications, including corticosteroids or cyclosporine A, within 30 days of beginning the study; current use of any oral antihistamines or anti-inflammatory medications; active ocular infection, ocular disease (other than dry eye syndrome), or uncontrolled systemic disease; any change in contact lens type, solutions or rewetting drops within the past 30 days; history of adverse reaction to either of the study medications; and prior history of corneal surgery. 
Use of artificial tears for dry eye management and contact lens wear were not considered to be inclusion or exclusion criteria. However, subjects who used tears or wore contact lenses were instructed to maintain their normal regimens throughout the study.

Once referred to the study, informed consent was obtained and subjects were randomized, using a random number generator, to one of two groups. Both groups were given a prescription-only medical food supplement, Tears Again HYDRATE $^{\mathrm{TM}}$ (OCuSOFT, Rosenberg, TX), consisting of four geltabs containing a total of $1000 \mathrm{mg}$ of omega-3 EFAs derived from flaxseed oil and $500 \mathrm{mg}$ of omega-6 EFAs (GLA) derived from evening primrose oil, both of which are microencapsulated in a proprietary liposome process designed to improve absorption. The amount of EFAs per food supplement serving is similar to that used in other research on evening primrose oil ${ }^{16}$ and identical to that used in other research on flaxseed oil. ${ }^{11}$

Initially, 56 subjects were enrolled. A total of 13 subjects dropped out of the study, only three of whom dropped out for reasons potentially related to the treatment. The reasons for dropout are identified in Table 1. Results were compiled for the remaining 43 subjects.

Group $1(n=23)$ was assigned to take two soft geltabs of the medical food supplement by mouth twice daily (four geltabs per day in all) for 6 months. Group $2(n=20)$ was directed to take the medical food supplement in the same manner, along with topical cyclosporine, instilled twice daily during the last 3 months of the study. The prescription food supplement was provided free of charge, and subjects were reimbursed for their purchase of topical cyclosporine. The study was compliant with Health Insurance Portability and Accountability Act privacy regulations.

Subjects in both groups were evaluated at baseline, month 1 , month 3 , and month 6 . Primary outcome measures included TBUT, conjunctival staining, corneal staining, and change in subjective symptoms. The clinicians evaluating

Table I Reasons for dropout

\begin{tabular}{ll}
\hline Reason & Number of subjects \\
\hline Poor compliance & 6 \\
Moved from area & 2 \\
Unrelated medical problems & 2 \\
(back injury, sinus infection) & \\
Upset stomach & $\mathrm{I}$ \\
Headaches & $\mathrm{I}$ \\
Incompatibility of flaxseed oil with & $\mathrm{I}$ \\
blood-thinner medication & \\
Total & 13 \\
\hline
\end{tabular}

the outcome measures were not masked to the cyclosporine group. Visual acuity testing and refractions were performed at each visit. The subjective assessment of change in symptoms was administered at 6 months. In this subjective assessment, patients were asked whether they experienced worsening or no improvement in symptoms, some improvement, or moderate to marked improvement.

A corneal staining evaluation was performed 1 minute after wetting a strip with $1 \mathrm{mg}$ of fluorescein dye and placing it into the eye. A cobalt blue filter on the slit lamp was used to visualize the staining pattern and tear breakup. Corneal staining was graded on a scale of $0-4$. Grade 0 was considered 0-2 punctate erosions in any two quadrants or less; Grade 1 was superficial punctate erosions in two or more quadrants, or centrally that could be individually counted (minute/fine point erosions with defined borders, faint fluorescence or minimal scattered stippling); Grade 2 was larger punctate erosions with diffuse borders in one or more quadrants (bright fluorescence or moderate stippling, increased density and coverage); Grade 3 was considered confluence of the punctate erosions in one or more quadrants or centrally; Grade 4 was confluence of erosions in the central cornea or involving 50\% or more of any quadrant.

Following the instillation of fluorescein dye, TBUT was defined as the first significant break in the fluorescein staining pattern of the tear film and was measured three times at every visit. TBUT was calculated using a stopwatch for a minimum of 2 minutes to a maximum of 3 minutes.

Conjunctival staining was performed 2 minutes after instilling a strip containing $1.5 \mathrm{mg}$ of lissamine green dye into each eye, and graded on a scale of $0-4$. Grade 0 was 0-2 punctate erosions in up to two quadrants; Grade 1 was punctate erosions in one or more quadrants ( $>2$ punctate erosions); Grade 2 was moderate punctate erosions in one or more quadrants ( $>20$ individually counted erosions); Grade 3 was confluence of the erosions in one or more quadrants (clumped punctuate erosions making it difficult to identify individual erosions); and Grade 4 was severe punctate erosions in one or more quadrants affecting $>50 \%$ of any quadrant.

A two-tailed, paired $t$-test was used to determine whether there was a significant difference $(P<0.05)$ between baseline and month 6 of the study for each of the two groups and whether there was any significant difference between the groups at month 6 .

\section{Results}

Table 2 lists the baseline, month 3, and month 6 results for the primary outcome measures. Both groups had a 
Table 2 Change in main outcome measures

\begin{tabular}{|c|c|c|c|c|c|c|}
\hline & & $\begin{array}{l}\text { Group I: } \\
\text { supplement only } \\
(n=23)\end{array}$ & $P$ value & $\begin{array}{l}\text { Group 2: } \\
\text { supplement }+ \\
\text { cyclosporine } \\
(n=20)\end{array}$ & $P$ value & $\begin{array}{l}\text { Between-group } \\
P \text { value }\end{array}$ \\
\hline TBUT & Baseline & 0.652 & & 0.583 & & 0.838 \\
\hline \multirow[t]{4}{*}{ (seconds) } & Month 3 & 1.196 & 0.231 & 1.422 & 0.021 & 0.760 \\
\hline & Month 6 & $\mathrm{I} .457$ & 0.558 & 1.590 & 0.653 & 0.780 \\
\hline & $\Delta$ baseline to & 0.805 & 0.006 & 1.007 & 0.004 & \\
\hline & 6 months & & & & & \\
\hline Corneal staining & Baseline & 0.848 & & 0.875 & & 0.893 \\
\hline \multirow[t]{4}{*}{ (0-4 scale) } & Month 3 & 0.739 & 0.328 & 0.650 & 0.165 & 0.700 \\
\hline & Month 6 & 0.717 & 0.833 & 0.675 & 0.825 & 0.859 \\
\hline & $\Delta$ baseline to & -0.131 & 0.299 & -0.200 & 0.248 & \\
\hline & 6 months & & & & & \\
\hline Conjunctival staining & Baseline & 1.130 & & 1.300 & & 0.548 \\
\hline \multirow[t]{4}{*}{ (0-4 scale) } & Month 3 & 1.261 & 0.514 & 1.300 & 1.000 & 0.879 \\
\hline & Month 6 & 1.391 & 0.492 & 1.575 & 0.142 & 0.487 \\
\hline & $\Delta$ baseline to & 0.261 & 0.218 & 0.275 & 0.326 & \\
\hline & 6 months & & & & & \\
\hline
\end{tabular}

Note: The only statistically significant changes were the changes in tear breakup time in both groups from baseline to 6 months.

Abbreviation: TBUT, tear breakup time.

statistically significant improvement in TBUT between baseline and month 6 . In the food supplement only group, TBUT improved by 0.805 seconds from baseline to month 6 . In the supplement/cyclosporine group, TBUT improved by 1.007 seconds from baseline. (Figure 1) There was no statistically significant difference in TBUT between the two groups at baseline, at month 3 (before the addition of topical cyclosporine therapy in group 2), or at the conclusion of the study at month 6 . There were no significant differences in corneal or conjunctival staining between groups or within a group during the course of the study.

In their subjective responses, approximately half the subjects in both groups reported moderate to marked improvement of symptoms between baseline and 6 months (Table 3 ).

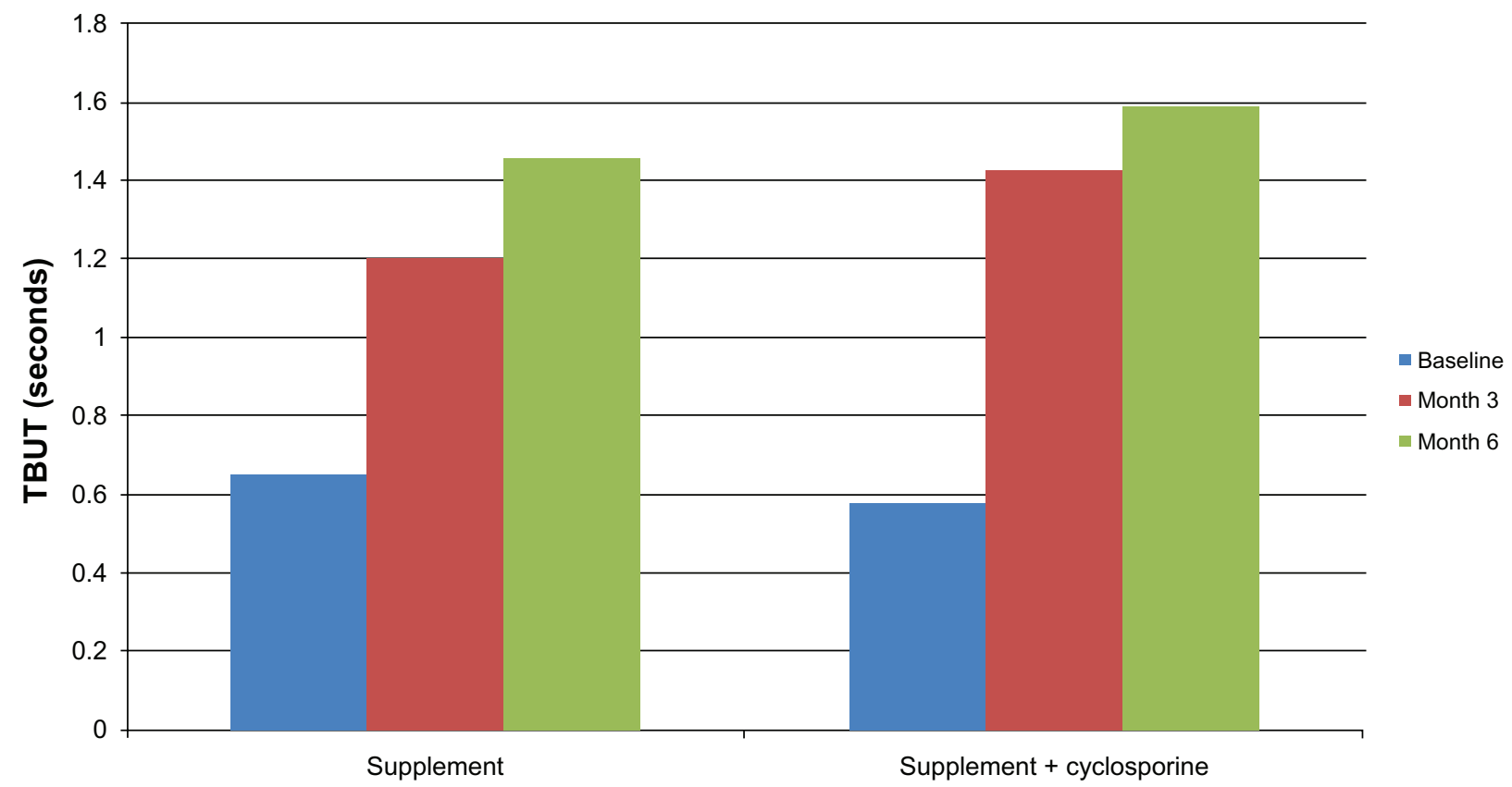

Figure I Improvement in TBUT (in seconds). Both groups had a similar and statistically significant improvement in TBUT from baseline to month 6 . There is no statistically significant difference between the groups at any point (baseline, month 3 , or month 6).

Abbreviation: TBUT, tear breakup time. 
Table 3 Subjective response of patients at 6 months regarding change in symptoms compared with baseline

\begin{tabular}{lll}
\hline & $\begin{array}{l}\text { Group I: } \\
\text { supplement only } \\
(\mathbf{n}=\mathbf{2 4})\end{array}$ & $\begin{array}{l}\text { Group 2: } \\
\text { supplement + } \\
\text { cyclosporine } \\
(\mathbf{n}=\mathbf{1 9})\end{array}$ \\
\hline $\begin{array}{l}\text { Worse symptoms or } \\
\text { no improvement }\end{array}$ & $17.4 \%$ & $15 \%$ \\
$\begin{array}{l}\text { Some improvement } \\
\text { Moderate to marked }\end{array}$ & $30.4 \%$ & $25 \%$ \\
improvement & $52.2 \%$ & $50 \%$ \\
\hline
\end{tabular}

\section{Discussion}

These findings suggest that the medical food supplement alone improved TBUT and relieved patient symptoms. The addition of a commonly used therapy, topical cyclosporine, did not convey any statistically significant improvement in TBUT beyond that achieved by the supplement, at least during the 3 -month period that group 2 used the Restasis drops.

Use of contact lenses, contact lens solution interactions, and artificial tear use patterns could be confounding factors. These were permitted during the study but not specifically analyzed, so it is not known whether there were any differences between the two groups or within a group in regard to contact lens wear or artificial tear usage.

The initial TBUT values in this study were quite low. Even after the modest improvement found in the study, the resulting TBUT values are still indicative of tear film instability. It is unknown whether measurement error contributed to this improvement. Nevertheless, the fact that TBUT improvement was accompanied by subjective symptom improvement suggests that the change in TBUT was clinically meaningful.

Some studies have suggested that the TBUT testing that is typically performed in clinical settings is not highly correlated with dry eye disease severity. ${ }^{20}$ TBUT is considered a significant sign in the ITF classification scheme for dry eye. ${ }^{3}$ New diagnostic tools that are under investigation or in the process of being launched commercially may provide additional insight into the effective management of dry eye. However, at the time of this study - and until such tools become widely available - TBUT is a reasonable tool for assessing dry eye in the clinical setting.

We believe there is sufficient evidence in the literature to suggest that the proper balance of omega-3 EFAs and the GLA form of omega- 6 EFAs should play a role in the treatment of dry eye. More research remains to be done on the composition and bioavailability of EFA medical food supplements like the one studied here in order to confirm their role in the treatment of dry eye. A future masked, well controlled, prospective study would be useful to confirm our results.

\section{Acknowledgments}

Funding for the study was provided by CYNACON/ OCuSOFT. The authors wish to acknowledge the editorial assistance of Jan Beiting of Wordsmith Consulting in drafting and styling the manuscript. Financial support for the editorial services was provided by OCuSOFT.

\section{Disclosure}

Dr Jackson serves on the Allergan speakers bureau and is a consultant to CYNACON/OCuSOFT. Dr Burrell has received research funding from Alcon, CYNACON/OCuSOFT, GlaxoSmithKline, and Pfizer. Dr Gaddie is a consultant to Allergan and CYNACON/OCuSOFT. Dr Richardson is an employee of CYNACON/OCuSOFT.

\section{References}

1. The epidemiology of dry eye disease: report of the Epidemiology Subcommittee of the International Dry Eye Work Shop (2007). Ocul Surf. 2007;5:93-107.

2. Lemp MA. Advances in understanding and managing dry eye disease. Am J Ophthalmol 2008;146:350-356.

3. Behrens A, Doyle JJ, Stern L, et al. Dysfunctional tear syndrome: a Delphi approach to treatment recommendations. Cornea. 2006;25: 900-907.

4. Management and therapy of dry eye disease: report of the Management and Therapy Subcommittee of the International Dry Eye Work Shop (2007). Ocul Surf. 2007;5:163-178.

5. Sall K, Stevenson OD, Mundorf TK, Reis BL. Two multicenter, randomized studies of the efficacy and safety of cyclosporine ophthalmic emulsion in moderate to severe dry eye disease. Ophthalmology. 2000;107:631-639.

6. Rosenberg ES, Asbell PA. Essential fatty acids in the treatment of dry eye. Ocul Surf. 2010;8:18-28.

7. SanGiovanni JP, Chew EY, Clemons TE, et al; and the Age-Related Eye Disease Study Research Group. The relationship of dietary lipid intake and age-related macular degeneration in a case-control study: AREDS Report No. 20. Arch Ophthalmol. 2007;125:671-679.

8. Miljanović B, Trivedi KA, Dana MR, et al. Relation between dietary n-3 and n-6 fatty acids and clinically diagnosed dry eye syndrome in women. Am J Clin Nutr. 2005;82:887-893.

9. Cakiner-Egilmez T. Omega-3 fatty acids and the eye. Insight. 2008;33: 20-25.

10. Roncone M, Bartlett H, Eperjesi F. Essential fatty acids for dry eye: a review. Cont Lens Anterior Eye. 2010;33:49-54.

11. Pinheiro MN Jr, dos Santos PM, dos Santos RC, et al. Oral flaxseed oil (Linum usitatissimum) in the treatment for dry-eye Sjögren's syndrome patients. Arq Bras Oftalmol. 2007;70:649-655.

12. Wojtowicz JC, Butovich I, Uchiyama E, et al. Pilot, prospective, randomized, double-masked, placebo-controlled clinical trial of an omega-3 supplement for dry eye. Cornea. 2011;30:308-314.

13. Schnebelen C, Viau S, Grégoire S, et al. Nutrition for the eye: Different susceptibility of the retina and the lacrimal gland to dietary omega-6 and omega-3 polyunsaturated fatty acid incorporation. Ophthalmic Res. 2009;41:216-224. 
14. Viau S, Maire MA, Pasquis B, et al. Efficacy of a 2-month dietary supplementation with polyunsaturated fatty acids in dry eye induced by scopolamine in a rat model. Graefes Arch Clin Exp Ophthalmol. 2009;247:1039-1050.

15. Belch JJ, Hill A. Evening primrose oil and borage oil in rheumatologic conditions. Am J Clin Nutr. 2000;71(Suppl 1):S352-S356.

16. Kokke KH, Morris JA, Lawrenson JG. Oral omega-6 essential fatty acid treatment in contact lens associated dry eye. Cont Lens Anterior Eye. 2008;31:141-146.

17. Pinna A, Piccinini P, Carta F. Effect of oral linoleic and gamma-linolenic acid on meibomian gland dysfunction. Cornea. 2007;26:260-264.
18. Aragona P, Bucolo C, Spinella R, et al. Systemic omega-6 fatty acid treatment and pge 1 tear content in Sjögren's syndrome patients. Invest Ophthalmol Vis Sci. 2005;46:4474-4479.

19. Barabino S, Rolando M, Camicione P, et al. Systemic linoleic and gamma-linolenic acid therapy in dry eye syndrome with an inflammatory component. Cornea. 2003;22:97-101.

20. Sullivan BD, Whitmer D, Nichols KK, et al. An objective approach to dry eye disease severity. Invest Ophthalmol Vis Sci. 2010;51:6125-6130.
Clinical Ophthalmology

\section{Publish your work in this journal}

Clinical Ophthalmology is an international, peer-reviewed journal covering all subspecialties within ophthalmology. Key topics include: Optometry; Visual science; Pharmacology and drug therapy in eye diseases; Basic Sciences; Primary and Secondary eye care; Patient Safety and Quality of Care Improvements. This journal is indexed on

\section{Dovepress}

PubMed Central and CAS, and is the official journal of The Society of Clinical Ophthalmology (SCO). The manuscript management system is completely online and includes a very quick and fair peer-review system, which is all easy to use. Visit http://www.dovepress.com/ testimonials.php to read real quotes from published authors. 